\title{
L'expérience architecturale
}

Architectural Experience

\section{Xavier Bonnaud}

\section{OpenEdition}

Journals

Édition électronique

URL : http://journals.openedition.org/crau/569

DOI : $10.4000 /$ crau.569

ISSN : 2547-5746

\section{Éditeur}

Éditions du patrimoine

\section{Édition imprimée}

Date de publication : 1 novembre 2012

Pagination : 158-164

ISBN : 978-2-7577-0108-9

ISSN : 1296-4077

Référence électronique

Xavier Bonnaud, «L'expérience architecturale», Les Cahiers de la recherche architecturale et urbaine [En ligne], 26/27 | 2012, mis en ligne le 01 novembre 2017, consulté le 20 avril 2019. URL : http:// journals.openedition.org/crau/569; DOI : 10.4000/crau.569 
Interroger l'expérience architecturale permet de mettre en évidence une des spécificités de l'architecture, sa puissance de modélisation, avec la richesse multidimensionnelle des situations qu'elle offre. Cette notion constitue le fil conducteur d'un faisceau de données et d'interrogations qui, un peu au-delà d'une attitude de recherche assise sur la stricte dissociation mécaniste, oblige à penser la complexité de l'entremêlement entre le chercheur et la connaissance qu'il s'attache à mettre en relief.

\section{L'expérience architecturale}

XAVIER BONNAUD

Point de vue sur une discipline et délimitation d'un champ de recherches

Dans le cadre d'une habilitation à diriger les recherches, nous portons actuellement au sein du GERPHAU, un travail de recherche et de prospection sur la notion d'expérience architecturale. Nous allons présenter ici succinctement les thématiques et les registres d'interrogations que cette notion permet d'aborder :

- elle questionne autant la réception de l'architecture (les conditions de sa rencontre, sa perception, les manières d'en parler, d'en débattre, de la théoriser), que ses savoirs de conception ;

- elle permet d'approfondir un champ philosophique particulier (la phénoménologie), dont les croisements avec l'architecture sont particulièrement féconds ;

- elle scrute le présent de notre société à partir des lieux où se recomposent l'expérience contemporaine du monde, avec une grande capacité à révéler les caractéristiques de notre époque ;

- elle met en évidence une des spécificités de l'architecture, sa 
puissance de modélisation et la richesse multidimensionnelle des situations qu'elle offre.

Cette notion constitue donc le fil conducteur d'un faisceau d'interrogations sur l'architecture. Différemment d'une attitude de recherche assise sur la stricte dissociation mécaniste, l'ouverture existentielle de l'expérience oblige à penser la complexité de l'entremêlement entre le chercheur et la connaissance qu'il s'attache à mettre en relief. Mais ce faisant, l'engagement dans le concret des lieux et des œuvres fait émerger la diversité et la richesse et des savoirs de position et de situation qui font la spécificité de la compétence architecturale. Cette notion ouvre des points de vue nouveaux sur la discipline et permet, comme depuis une table d'orientation bien située, d'identifier des repères marquants et d'interroger les horizons plus indéfinis. Elle participe à un arpentage toujours en cours des savoirs de la discipline et offre sur de nombreuses possibilités de recherches. Regardons de manière concise les thèmes et problématiques qu'ouvre cette notion.

\section{L'expérience contemporaine du monde}

En ce début de $x \mathrm{XI}^{\mathrm{e}}$ siècle, notre expérience du monde s'inscrit encore dans la continuité de l'aventure moderne et de ces grandes tendances (mécanisation, individualisation, accélération, urbanisation), auxquelles s'ajoute l'artificialisation ${ }^{1}$. Mais à regarder la multiplication et l'imbrication actuelles des crises (financières, économiques, climatiques, sociales, culturelles, énergétiques), émerge l'impression bizarre que la déraison s'est emparée du monde. Il semble que l'imaginaire de la catastrophe influence les esprits et accompagne la conscience de la mondialisation; il semble que depuis la victoire idéologique du libéralisme, l'impuissance politique domine la pensée contemporaine.

La confusion qui s'opère entre buzz, informations, connaissances, pensée, diffuse un brouillage dans notre relation au réel. La publicité, les médias, la communication politique déploient des stratégies " présentielles » qui bombardent le quotidien des milliards de citadins que nous sommes. Un bruit de fond continu sature l'espace public et l'horizon mental de chacun, transformant en chemin notre assise dans le monde. Walter Benjamin parlait "d'atrophie de l'expérience »: cette " télémécanisation " de notre relation aux choses semble parfois lui donner raison.

C'est dans le champ de turbulences de ce début de siècle que prend corps la grande diversité des productions architecturales et urbaines. Certaines exacerbent la virtuosité opératoire contemporaine et l'esthétique de la mondialisation qui va avec. Elles nous en présentent les inédites potentialités, jouant d'une formidable inventivité technique comme du consumérisme planétaire pour proposer une jubilation de la performance et du plaisir du signe partagé. D'autres au contraire, moins nombreuses, se concentrent sur des valeurs plus intemporelles, celles de la matière, de la lumière, du corps, de la communauté, d'une économie dans l'emploi de ressources locales, d'une éthique constructive qui cherche de nouveaux contacts avec la nature et ses éléments.

Toujours est-il que, sous les coups de boutoir de l'idéologie libérale, reste en vrac le chantier d'une

1. Xavier Bonnaud, La technocité, devenir urbain de l'humanité ?, thèse d'urbanisme, IUP, université Paris XII-Créteil, 2005 et De la ville au technocosme, Nantes, Éditions l'Atalante, 2008. 
philosophie de l'aménagement dont on imagine que I'architecture puisse en être l'expression culturelle la plus avancée. Un modèle de développement soumis à un rationalisme étriqué lutte contre l'émergence de paradigmes environnementaux. Bien qu'ils peinent à s'énoncer, c'est à leur définition que nous souhaitons contribuer.

\section{Architecture et phénoménologie}

La notion d'expérience possède à la fois une polysémie contemporaine stimulante et un passé riche. À différentes époques, des traditions philosophiques se sont interrogées sur le statut de l'expérience, sur son rôle fondamental dans notre capacité à fonder toute connaissance, sur sa subjectivité, ses dimensions multiples. Elle interroge notre relation au réel et met en dialogue des courants de pensées comme le rationalisme, l'empirisme, le subjectivisme, le naturalisme, le mysticisme, l'individualisme... Elle ouvre plusieurs catégories de discours, narratifs, interprétatifs, psychologiques, cognitivistes, poétiques, culturalistes, symboliques ...

Au $x x^{e}$ siècle, l'approfondissement philosophique de la notion d'expérience a pris un nouvel essor avec l'approche phénoménologique. Des champs de connaissances et de réflexions stimulants se sont déployés à partir de travaux sur la perception et le corps ${ }^{2}$. L'esthétique ${ }^{3}$ aussi a été dynamisée par la phénoménologie qui a permis de décrire et d'élargir la « rencontre artistique » à toutes les palettes de l'aventure sensorielle et sensible, bien au-delà des domaines du visible et de la représentation dans lesquels, en France, une tradition beaux-arts bien ancrée la cantonnait.
Revisiter cette notion d'expérience et des multiples éléments qui en constituent la richesse construit un domaine de recherche par nature interdisciplinaire. Fluide et multiple, cette notion partage avec l'architecture un caractère multidimensionnel, (un environnement culturel, un cadre physique, un temps de réception oscillant entre perception, projection, élaboration), et produit simultanément " du sens et du sensible 4 ».

Ayant à l'esprit la manière très claire dont Erwin Panofsky a montré en quoi la structure des cathédrales rendait concret le contemporain de la pensée scolastique $^{5}$, nos interrogations sur la notion d'expérience posent un regard contemporain sur l'architecture. II s'agit de décrypter les modes de percevoir, les formes mentales, les imaginaires poétiques et politiques qui constituent le soubassement de ce siècle qui commence. Fuvres, situations construites, événements architecturaux et urbains ouvrent à des interprétations esthétiques, fonctionnelles, sociales, ou politiques. Mais à côté de ces regards fragmentés, la richesse du fait architectural, sa capacité de construction concrète, son " effet de présence ${ }^{6}$, permettent $d^{\prime}$ interroger de manière très directe les instruments perceptifs et intellectuels de notre époque. La formidable capacité de modelage et d'enveloppement de l'architecture, comme le fait que nous en soyons les " prisonniers volontaires", participe puissamment « de notre relation au réel, de sa description et en fin de compte de sa définition ${ }^{7} . »$

C'est en ce sens que la notion d'expérience architecturale est riche : elle interpelle avec une particulière efficacité notre rapport au réel. Elle engage le visiteur, I'habitant, le promeneur, le citadin et interroge des
2. Voir particulièrement l'ensemble de I'œuvre de Maurice Merleau-Ponty.

3. Avec les travaux de Mikel Dufrenne et son ouvrage Phénoménologie de l'expérience esthétique, Paris, PUF, 1953 et sous son impulsion l'influence de la revue Esthétique.
4. Jean Nouvel, Leçon inaugurale de l'École de Chaillot, prononcé le 8 janvier 2008, co-édition, Silvana Editoriale, Cité de l'architecture et du patrimoine, 2009.

5. Erwin Panofsky, Architecture gothique et pensée scolastique, Paris, Éditions de Minuit, 1967.
6. Définition que donne Christian de Portzamparc de l'architecture dans Voir écrire, ouvrage rapportant un ensemble de discussions avec Philippe Sollers, (Paris, Calmann-Lévy, 2003).

7. Alain Corboz, «Comment penser la mutation ", Sortons enfin du labyrinthe, Gollion, Infolio, 2009 
parcelles de sens agglomérées, parfois explicites parfois énigmatiques mais qui, mises bout à bout, dessinent le creuset d'une époque, son imaginaire, voire son épistémè. Par son caractère fluide, elle permet de réfléchir aux mutations en cours, elle met en chantier les paradigmes. De plus, elle n'est pas surdéterminée par une forme architecturale et ne peut être instrumentalisée par un quelconque style.

S'ouvrir à la dimension paradoxale de l'expérience architecturale, c'est accepter la transformation de notre conception du monde et assumer qu'une certaine complexité ne puisse être réduite. C'est s'engager à visiter des frontières floues à partir d'une mesure humaine.

\section{Corps et expérience}

L'œuvre de Maurice Merleau-Ponty a formidablement décrypté le rôle du sensible et du corps dans l'expérience humaine. À la prétendue objectivité de la science, il n'a cessé de formuler " une expérience du monde, un contact avec le monde qui précède toute pensée sur le monde ${ }^{8}$ ".

Il semble que l'actuelle crise environnementale fonctionne comme une entrée dans ce que le géographe Jacques Levy nomme "I'ère des environnements ». En effet, n'est-ce pas face au dérèglement climatique qu'émerge l'interaction entre le tout et la partie ? N'est-ce pas en regardant la biodiversité s'effondrer qu'il nous faut considérer la rétroaction permanente entre ce qui contient et ce qui est dedans? Nous pénétrons dans une époque réflexive qui nous demande de prendre en compte la double dépendance englobant/englobé. De ces constats émerge une

8. Maurice Merleau-Ponty, Sens et non-sens, Paris, Gallimard [1996]. sensibilité nouvelle, se déploie une conscience environnementale qui dépasse les précédentes représentations mécanistes pour fonctionner aussi comme "empathie environnementale ». Merleau-Ponty n'a cessé de parler de la présence de cette assise primordiale dans le monde, d'œuvrer à une "réhabilitation ontologique du sensible " dont on perçoit l'urgence actuelle pour simplement sentir ce que l'on sait des dysfonctionnements environnementaux.

À côté des recherches à visée scientifique sur les ambiances et des apports de la psychologie environnementale, il est indispensable d'interroger philosophiquement notre relation aux environnements. La notion d'expérience architecturale y participe de manière forte : elle met étroitement en relation architecture et phénoménologie et permet d'approfondir ce que Steven Holl nomme la richesse de l'entremêlement permanent entre les hommes et leurs environnements construits, ce jeu de coulisses et d'imbrications continuelles entre arrière-fonds, horizons intermédiaires et environnements immédiats ${ }^{9}$. L'interrogation phénoménologique (comme philosophie de l'entremêlement) offre des pistes précieuses pour approfondir de telles thématiques. Elle offre aussi des pistes pour les relier à la dynamique de globalisation, à la disparition d'une certaine extériorité comme aux tensions actuelles entre englobant et englobé (l'empreinte écologique des établissements humains), afin de mettre en débat les nouvelles relations à la planète et à l'environnement que nous avons à construire.

Prenons la question par une autre entrée. En observant le déploiement des technologies numériques
9. Steven Holl, «Intertwinning », El Croquis $n^{\circ}$ 93, 1996 et aussi Questions of Perception: Phenomenology of Architecture, San Francisco, William Stout Publishers, 2006. 
et l'investissement massif dans le virtuel concomitant aux dérèglements du monde physique, on ressent le besoin d'une philosophie (puis d'une culture et de savoir-faire) qui permettent de reconstruire une amitié avec le monde physique. La quête de la réalité augmentée (qui superpose au réel des contenus non perceptibles et non physiquement co-présents) ne doit pas rester l'apanage des nouvelles technologies numériques. Sur une base intensément physique, hébergeant et magnifiant la dimension concrète de notre condition humaine ${ }^{10}$, l'architecture offre aussi une réalité augmentée. Elle propose des myriades d'expériences, mais des expériences ouvertes à tous, libres de droit, indépendantes des langues et des niveaux de vies, et qui de plus mettent en jeu culturellement et anthropologiquement notre rapport à la nature, aux éléments, au monde, au corps, aux autres.

Mais cela ne peut se construire que dans un dialogue serré et exigeant avec la puissance de destruction/ création qu'à ouvert la dynamique du capitalisme. Cela ne peut s'étudier précisément que par la rencontre attentive avec des œuvres et le détail des expériences qu'elles proposent.

\section{Richesses et diversités de l'expérience architecturale}

À partir d'œuvres contemporaines, bien des recherches peuvent se construire sur le décryptage des spécificités de l'expérience architecturale. Car les œuvres les plus fortes fonctionnent comme des sismographes : elles rendent lisibles des micro-tremblements qui annoncent le déplacement de plaques plus importantes. Leur étude concrète reste le terrain indispensable des thèses en architecture.

Déchiffrer les expériences architecturales demande de réfléchir aux critères de la réception et d'élargir la palette sensorielle à partir de laquelle se construit I'appréciation architecturale. II convient dépasser ce que I'architecte finlandais Juhani Pallasmaa ${ }^{11}$ nomme "l'occulocentrisme occidental ", cette domination de la vision sur la pensée qui désensibilise d'autres canaux et produits les dérives du narcissisme des objets et du nihilisme par l'effacement de la dimension charnelle de notre assise dans le monde. Les canaux sensoriels sont bien plus nombreux que les cinq sens traditionnels, et cet élargissement multisensoriel de la perception des environnements peut être travaillé à partir des bases scientifiques très concrètes énoncées par Alain Berthoz sur la physiologie de la perception et de l'action.

Pour aborder la production contemporaine, plusieurs pistes sont ouvertes. On peut aborder la variété des architectures contemporaines à partir des différentes modalités sensorielles qu'elles révèlent. On peut aussi s'adosser à la classification vitruvienne pour interroger de biais les registres d'expériences.

Parfois est mise en avant l'expérience de la construction. La matérialité des bâtiments et l'éclat que leur structure s'impose. La puissance, la clarté des objets architecturaux, leur solidité intrinsèque et leur ingéniosité technique dominent. Parfois l'expérience se fait aventure sociale et plaisir des usages. Une relation forte se tisse entre les édifices et les communautés humaines qu'ils hébergent, influençant appréciations et représen-
10. André Gorz, L'immatériel, Paris, Galilée,

2003.
11. Juhani Pallasmaa, The Eyes of the Skin, The Architecture and the Senses, Chichester, John Wiley \& Sons, 2005. 
tations des lieux. Parfois l'expérience architecturale est avant tout subjective, émotionnelle, esthétique, elle prend des colorations personnelles, intensifie les arrières plans psychologiques.

On peut encore, sous un autre angle, aborder les modes de percevoir qui, tantôt rationnels, tantôt relationnels, tantôt sensibles, engagent chacun différentes catégories d'appréciation et de discours. Nous proposons à titre d'illustration quatre/cinq grands registres d'expériences comme autant de thèmes dynamiques qui irriguent la conception architecturale ${ }^{12}$.

- La sensorialité. Une exacerbation sensorielle est aujourd'hui à la fois proposée et attendue. Fonctionnant comme perturbation des déterminants de base de l'expérience architecturale (la gravité l'orthogonalité, la déformation, la désorientation spatiale ...), ou par l'envahissement d'un registre contre tous les autres (l'intensité chromatique, l'indéfinition, la sensualité des matières ...). Ce déploiement de l'accroche sensorielle s'enracine dans l'histoire moderne et post-moderne de I'architecture. Cette tendance fait écho à une revendication du type "Je ressens donc je suis », qui traverse notre époque : elle interroge évidemment le processus réceptif.

- L'échelle. De nombreuses constructions nous confrontent au gigantisme. La question du dimensionnement traverse I'histoire de l'architecture dans sa relation avec les pouvoirs, dans son désir de monumentalité. Mais la puissance de modelage grandit avec I'habileté technique et des œuvres chaque jour plus nombreuses revendiquent cet horizon du gigantisme qu'exige la scène économique mondiale et sa guerre des "visibilités ". Ici se pose la question de l'emballement de notre imaginaire bâtisseur, d'un débordement opératoire indépendant du sens de la mesure.

- Le contexte. Bien des constructions proposent des expériences construites à partir de leur contexte, de l'ici et là des données terrestres, (l'horizon, du sol, la roche, la lumière naturelle, le paysage, la végétation ...). Elles fonctionnent comme révélation de ce qui est plutôt que recherche d'inédits. Ici, se regroupent des aspirations plus écocentrées, fonctionnant comme partage de situations quasi géographiques, comme témoignages, attention commune à la planète.

- L'intériorité. Des enseignes de bricolage ou d'ameublement, des magazines grand public parlent abondamment du bonheur qu'apporte directement l'aménagement des lieux. Les espaces plutôt domestiques sont présentés de manière libre à partir de plusieurs registres (la raison, le sentiment, l'intuition, la sensation, l'appropriation, le plaisir). Le bien-être qu'ils procurent, leur appropriation symbolique sont mis en évidence. Cette tendance fait écho au nihilisme de notre époque, à l'uniformité des standards de la globalisation et au caractère générique de trop nombreuses productions.

- La convivialité. Bien des œuvres trouvent leur saveur dans un temps commun de paix partagée, ouvrant une plénitude fugace. II s'agit de lieux qui valorisent la rencontre, des espaces soucieux du plaisir d'être ensemble, légèrement. S'y découvre l'ambivalence de notre époque entre peur, sécurisation et réinstitution d'un en-commun plus simple, plus accueillant.

12. X. Bonnaud, "Les contours d'une esthétique écologique », Traits urbains, $n^{\circ} 39$, juin 2010, p. 35-39. 


\section{Transmission et pédagogie}

Nous avons maintes fois constaté l'intérêt qu'il y a à aborder l'architecture, (sa richesse et son acuité à témoigner des enjeux contemporains) à partir de la notion d'expérience architecturale : cela permet de dialoguer avec un public plus large car cette notion est commune aux concepteurs et aux visiteurs. De nombreux travaux à visée didactique peuvent se déployer autant en direction des étudiants de licence que sous la forme d'ouvrages de vulgarisation vers un public de non-spécialistes. Ils s'inscrivent dans le prolongement des propos de Steen Eiler Rasmussen dans la présentation de son ouvrage, Experiencing $\operatorname{architecture}^{13}$ : "On ne produit pas de l'architecture en additionnant simplement des plans, les coupes les élévations. C'est quelque chose d'autre de plus. II est impossible d'expliquer précisément ce que c'est : ses limites ne sont clairement pas définies. En somme l'art ne saurait s'expliquer ; il doit s'éprouver. Mais avec des mots, il est possible d'aider les autres à en faire l'expérience ${ }^{14}$. " Notre connaissance autant de l'enseignement de l'architecture que des échanges de la sphère professionnelle nous laisse espérer de fructueuses perspectives dans ce domaine. Nombreux sont les adeptes d'une philosophie pragmatique qui se fient à leurs expériences tout en découvrant en chemin la richesse des contenus culturels, des possibilités d'échanges et de questionnements qu'offre l'architecture.

Voici donc rapidement présentés quelques thèmes regroupés autour de l'expérience architecturale. Au-delà des problématisations ici énoncées, cette notion permet aussi de donner vie à un corpus important de références architecturales, scientifiques et philosophiques dont nous avons déjà remarqué, lors des séminaires et colloques dans lesquels nous sommes engagés ${ }^{15}$, la curiosité qu'il suscite et les ouvertures bien concrètes qu'il offre vers de futurs doctorats en architecture.
13. Steen Eiler Rasmussen, Découvrir I'architecture, Paris, Le Linteau, 2002, pour la traduction française (introduction de Guy Desgrandchamps) ; Experiencing Architecture, Cambridge Mass., The MIT Press, 1959, pour la première édition en langue anglaise. Ce livre fait référence depuis sa parution dans les pays de langue anglaise auprès des étudiants de premier cycle.
14. Ibid, page 20 .

15. Dans les séminaires de master de I'option recherche et dans les colloques organisés chaque année: «Architecture et nature " en décembre 2009,

"Perception et architecture » en décembre 2010. "Architecture et frugalité » en décembre 2011. 